\title{
Authors' Reply to Cohen et al.'s Comment on “Central Demyelinating Diseases after Vaccination Against Hepatitis B Virus: A Disproportionality Analysis within the VAERS Database"
}

\author{
Julie Mouchet $^{1}$ (D) $\cdot$ Bernard Bégaud $^{1}$
}

Published online: 29 September 2018

(c) Springer Nature Switzerland AG 2018

We thank Cohen et al. for their interest in our paper "Central Demyelinating Diseases after Vaccination Against Hepatitis B Virus: A Disproportionality Analysis within the VAERS Database" [1]. As mentioned in this paper, our objective was to compare the frequency of reports of central demyelination [including multiple sclerosis (MS)] after anti-hepatitis B (HB) vaccination with other vaccinations. Disproportionality analyses (DPAs) aim at detecting signals within spontaneous reporting systems but they do not allow inferences to be drawn about the causal nature of an event-drug couple. In our case, stratifying DPAs on time period would have only led to distributing the risk across the period but would not have changed the main conclusions. That was therefore what was observed in the analysis by Cohen et al.

We found Table 1 very interesting in the comment by Cohen et al. Their results actually converge with ours. Their Table 1 showed significant ratios for foreign cases, which originated from outside of USA and presumably Europe. Ratios were clearly higher in the first period (1987-2000), a period corresponding to the launch of massive anti-HB vaccination campaigns in Europe. In France, mass immunization started in 1994 with the aim of targeting newborns and adolescents, in addition to at-risk adults. However, a complete disjunction was observed between the target and joint populations. Fewer than $20 \%$ of newborns were vaccinated while $\sim 21.6$ million adults received the HB vaccine at an age prone to developing central demyelination [2]. Two years after the launch of the vaccination campaign,

This reply refers to the comment available at https://doi. org/10.1007/s40264-018-0733-4.

Julie Mouchet

julie.le-moal@u-bordeaux.fr

1 Inserm, Bordeaux Population Health Research Centre, Team Pharmacoepidemiology, UMR 1219, University of Bordeaux, 33000 Bordeaux, France
249 cases of central demyelination occurring after HB vaccination were reported to the French pharmacovigilance (PV) system, leading to the initial signal [3]. The data reported by Cohen et al., stratified by period and origin of cases, well reflect this situation. Higher ratios were observed for foreign cases and in the oldest periods, supporting the hypothesis that DPA stratification led to the distribution of the risk across the period.

We totally agree that this signal was largely highlighted by the media, public attention peaking in 1998 and then decreasing over time. However, the cut-off chosen by Cohen et al. (i.e., 2004) seems surprising and difficult to justify. We do not see how a very limited number of French litigation cases may have influenced case reporting in USA. No publication supports the argument presented by Cohen et al. about the impact of these litigation cases on the reporting of MS either in France or USA. Media interest and litigation can of course increase reporting, i.e., by making under-reporting of associations, causal or fortuitous, less intense, which in any case exist. We also point out that the identification of French litigation cases is not possible in the Vaccine Adverse Event Reporting System and the number of these cases is doubtlessly quite small.

As reported by the European Medicines Agency in their recommendations on screening for adverse reactions in EudraVigilance, stratification is generally used in epidemiology to reduce bias due to confounding, when a third variable is associated both with the drug exposure and the event of interest. It may also be of benefit in signal detection algorithms [4]. In our case, litigation can be ruled out as a third variable that could impact the occurrence of central demyelination in a patient exposed or not to the HB vaccine. Furthermore, as mentioned by Cohen et al. in their comment, we followed the recommendations of the US Centers for Disease Control and Prevention regarding the stratification of DPAs by origin of cases (foreign vs. USA) [5]. 
We are pleased to note that our systematic literature review and meta-analysis [6] also caught the attention of Cohen et al. Both papers were submitted at the same time, thus it was difficult to cross-reference the papers. Nevertheless, we do not see why the findings of these two papers should be considered as contradictory. While our disproportionality analysis in the Vaccine Adverse Event Reporting System compared the frequency of reports between HB and other vaccinations without intending to assess causality, our meta-analysis pooled all estimates found in published studies assessing a potential link between HB and the occurrence of central demyelination. Our main conclusion was that the pooled estimates failed to demonstrate an association between the HB vaccine and central demyelination. Therefore, there are two explanations: either the link does not exist or the meta-analysis failed to reach statistical significance because of a lack of power. No firm conclusion should therefore be drawn.

We also underline the fact that the findings of Geier (2005) are consistent with our findings when comparing the frequency of case reports for autoimmune diseases including MS after $\mathrm{HB}$ vaccinations with tetanus vaccinations [7]. In their study, adults receiving the HB vaccine had a significantly increased odds ratio for MS $(5.2 ; 95 \%$ confidence interval $1.9-20 ; p<0.0003$ ), unlike the tetanus-containing vaccine-exposed group. Tetanus vaccines were chosen as a comparator given that they have never been suspected of a risk excess for central demyelination and were also administered to adults.

To conclude, it appears the analysis by Cohen et al. led to conclusions similar to ours, with all ROR produced in their analysis, except one ratio, being statistically significant. Moreover, some of their results were even marked as reporting odds ratios (ROR) for the HB vaccine after stratification on time and origin was higher for the oldest periods and foreign cases, peaking at ten.
Funding This reply was funded by the University of Bordeaux, France and the 'Institut national de la santé et de la recherche médicale' (Inserm). The funding source had no role in the study design, data collection and analysis, decision to publish, or preparation of this reply.

\section{Compliance with Ethical Standards}

Conflict of interest Julie Mouchet and Bernard Bégaud have no conflicts of interest that are directly relevant to the content of this reply.

\section{References}

1. Cohen C, Houdeau A, Khromava A. Comment on "Central Demyelinating Diseases after Vaccination Against Hepatitis B Virus: A Disproportionality Analysis within the VAERS Database. Drug Saf. 2018. https://doi.org/10.1007/s40264-018-0733-4.

2. Agence Française de sécurité sanitaire de produits de santé. Vaccination anti hépatite $\mathrm{B}$ : mise à jour des données et des études de pharmacovigilance. 2000. https://ansm.sante.fr/var/ansm_site/ storage/original/application/b460abed4a9a61d8dad78d43640333 54.pdf. Accessed 24 Sep 2018.

3. Fourrier A, Bégaud B, Alpérovitch A, Verdier-Taillefer MH, Touzé E, Decker N, et al. Hepatitis B vaccine and first episodes of central nervous system demyelinating disorders: a comparison between reported and expected number of cases. Br J Clin Pharmacol. 2001;51(5):489-90.

4. European Medicines Agency. Screening for adverse reactions in EudraVigilance. 2016. http://www.ema.europa.eu/docs/en_GB/ document_library/Other/2016/12/WC500218606.pdf. Accessed 24 Sep 2018.

5. US Centers for Disease Control and Prevention. Advisory guide to the interpretation of VAERS data. 2016. https://wonder.cdc.gov/ wonder/help/vaers/VAERS\%20Advisory\%20Guide.htm. Accessed 24 Sep 2018.

6. Mouchet J, Salvo F, Raschi E, Poluzzi E, Antonazzo IC, De Ponti $F$, et al. Hepatitis B vaccination and the putative risk of central demyelinating diseases: a systematic review and meta-analysis. Vaccine. 2018;36(12):1548-55.

7. Geier DA, Geier MR. A case-control study of serious autoimmune adverse events following hepatitis B immunization. Autoimmunity. 2005;38(4):295-301. 